\title{
THE BOUNDARY BEHAVIOR OF HOLOMORPHIC FUNCTIONS
}

\author{
STEVEN G. KRANTZ
}

\begin{abstract}
We study the boundary behavior of Hardy space functions on a domain in $\mathbb{C}^{n}$. We prove Fatou-type theorems using a new paradigm that avoids some of the difficult calculations in earlier work. Our results apply to a strictly broader class of domains.
\end{abstract}

1. Introduction. The study of the boundary behavior of holomorphic functions dates back to the work of Fatou in 1906. He proved that a bounded, holomorphic function on the disc has boundary limits almost everywhere through nontangential approach regions.

Later work extended Fatou's results to the $H^{p}$ spaces, $0<p<\infty$. The work of Littlewood and others showed that these results were sharp.

Beginning in 1969, Koranyi $[\mathbf{1 3 , 1 4}]$ and others showed us that the situation in several complex variables is quite different. For, on domains in $\mathbb{C}^{n}$ with $n>1$, we can allow the tangential boundary approach (at least in certain directions) for a Fatou-type theorem. Specifically, Koranyi showed that, on the unit ball $B$ in $\mathbb{C}^{n}$, we can use approach regions of the form

$$
\mathcal{A}_{\alpha}(P) \equiv\{z \in B:|1-z \cdot \bar{P}|<\alpha(1-|z|)\},
$$

$P \in \partial B, \alpha>1$, for the boundary limits in a Fatou theorem.

There has been great interest in the last 40 years in determining the sharp form of the Fatou theorem for a generic domain in $\mathbb{C}^{n}$. The monograph [7] will in fact tell the full story of this research program.

2010 AMS Mathematics subject classification. Primary 32A30, 32A35, 32A40.

Keywords and phrases. Holomorphic function, boundary limits, Fatou theorem, approach regions.

Received by the editors on November 13, 2012, and in revised form on October 4, 2013. 
Many of the results in several complex variables have been quite difficult and technical to prove. Thus, one finds it discouraging to attempt to consider more general contexts.

The purpose of the present work is to present a more abstract, softer approach to these matters. We can recover the results on domains of finite type in $\mathbb{C}^{2}$ and also present new results on more general domains in $\mathbb{C}^{n}$. The techniques here are very geometric and therefore appealing on philosophical grounds. They are inspired by ideas first presented in $[15,16]$ and also on an important but little-known covering theorem of Besicovitch (see [8, subsection 2.8.14] and [17]).

2. Spaces of homogeneous type. Our goal here is to formulate and prove a version of the Fatou theorem in $\mathbb{C}^{n}$ that avoids a lot of nasty calculation of the approach regions and the accompanying balls in the boundary. The methodology is rather abstract but can easily be specialized down to particular situations and particular domains. In this way, we recover the known results in the subject, and point to some new ones as well.

One of the main points of our construction is to render the boundary of a smoothly bounded, pseudoconvex domain in $\mathbb{C}^{n}$ as a space of homogeneous type in the sense of Coifman and Weiss [6]. This, of course, is an important device in the harmonic analysis of a space (see $[21])$.

Here it is worth reviewing what pseudoconvexity is. Let $\Omega \subseteq \mathbb{C}^{n}$ be a smoothly bounded domain, and let $\rho$ be a defining function for $\Omega$ (see [15]). Thus, $\Omega=\left\{z \in \mathbb{C}^{n}: \rho(z)<0\right\}$. If $P \in \partial \Omega$, then we say that $w \in \mathcal{T}_{P}(\partial \Omega)$ if

$$
\sum_{j=1}^{m} \frac{\partial \rho}{\partial z_{j}}(P) w_{j}=0 .
$$

This defines the complex tangent space to $\partial \Omega$ at $P$. We say that $\Omega$ is Levi pseudoconvex at $P$ if, for each $w \in \mathcal{T}_{P}(\partial \Omega)$, it holds that

$$
\sum_{j, k=1}^{n} \frac{\partial^{2} \rho}{\partial z_{j} \partial \bar{z}_{k}}(P) w_{j} \bar{w}_{k} \geq 0 .
$$

The domain is strongly pseudoconvex at $P$ if the inequality is strict for $w \neq 0$. 
In what follows, we shall be working with a fixed, smoothly bounded, Levi pseudoconvex domain $\Omega \subseteq \mathbb{C}^{n}$.

Recall that the Szegö kernel $S(z, \zeta)$ is the canonical reproducing kernel for the Hardy space $H^{2}(\Omega)$ (see [15, Chapter 1]). We will assume in what follows that $S$ extends to be continuous on $(\bar{\Omega} \times \bar{\Omega}) \backslash \triangle$, where $\triangle$ is the boundary diagonal. (Pseudolocality of the $\bar{\partial}_{b}$-Neumann problem is sufficient for this last condition to hold. Here pseudolocality simply means that $N_{b} \varphi$ is smooth where ever $\varphi$ is smooth. One simply applies the Kohn formula $P=I-\bar{\partial}_{b}^{*} N_{b} \bar{\partial}_{b}$ to the Dirac delta mass (see [9]). Here, $\bar{\partial}_{b}$ is the boundary version of the classical $\bar{\partial}$ operator. For the latter, see [15]. For the former, see $[4,9]$.)

Definition 2.1. Let $\Omega$ be as above and $P \in \partial \Omega$. Let $r>0$. For $P \in \partial \Omega$, define the ball as

$$
\beta(P, r)=\{\zeta \in \partial \Omega:|S(P, \zeta)|>1 / r\} \cup\{P\} .
$$

(The addition of $\{P\}$ here is somewhat redundant.)

If $\zeta^{1}, \zeta^{2}$ are points of $\partial \Omega$, then let us say the $\delta\left(\zeta^{1}, \zeta^{2}\right)<2 r$ if there is a ball $\beta(P, r)$, with $P \in \partial \Omega$, such that $\zeta^{1} \in \beta(P, r)$ and $\zeta^{2} \in \beta(P, r)$. We can define $\delta\left(\zeta^{1}, \zeta^{2}\right)$ by taking an obvious infimum.

If $\zeta^{1}$ and $\zeta^{2}$ are points of $\partial \Omega$, then a chain from $\zeta^{1}$ to $\zeta^{2}$ is a sequence of points $p^{0}, p^{1}, \ldots, p^{k}$ such that $p^{0}=\zeta^{1}$ and $p^{k}=\zeta^{2}$.

Definition 2.2. If $\zeta^{1}, \zeta^{2} \in \partial \Omega$, then we set

$$
d\left(\zeta^{1}, \zeta^{2}\right)=\inf \left\{\sum_{j=1}^{k} \delta\left(p^{j-1}, p^{j}\right):\left\{p^{j}\right\}_{j=0}^{k} \text { is a chain from } \zeta^{1} \text { to } \zeta^{2}\right\} \text {. }
$$

It is automatic that $d$ is a metric. In particular, the triangle inequality follows just from definition chasing.

Definition 2.3. If $P \in \partial \Omega$, then define the boundary ball

$$
B(P, r)=\{\zeta \in \partial \Omega: d(P, \zeta)<r\}
$$

for $r>0$. 
Recall now [6] the concept of a space of homogeneous type:

Definition 2.4. Let $X$ be a topological space equipped with a collection of balls $B(P, r)$ and a measure $\mu$. Here, $P \in X$ and $0<r<\infty$. Assume that each $B(P, r)$ is an open set. We say that $X$ is a space of homogeneous type if:

(a) $0<\mu(B(P, r))<\infty$ for every $P \in X$ and $r>0$;

(b) there is a $C_{1}>0$ so that $\mu(B(P, 2 r)) \leq C_{1} \cdot \mu(B(P, r))$ for every $P \in X$ and $r>0$ (this is sometimes called the doubling property);

(c) there is a $C_{2}>0$ so that if $B(Q, s) \cap B(P, r) \neq \emptyset$ and $s \geq r$. Then $B\left(Q, C_{2} s\right) \supseteq B(P, r)$ (this is sometimes called the enveloping property).

Equipping $\partial \Omega$ as above with surface measure $\sigma$ (i.e., $(2 n-1)$ dimensional Hausdorff measure), we wish to claim that $\partial \Omega$ with the balls $B(P, r)$ forms a space of homogeneous type. Property (a) is obvious, as any open set in $\partial \Omega$ has positive, finite measure. Property (c) is also clear, because the balls $B(P, r)$ come from a metric (just use the triangle inequality). Verification of property (b) requires a bit of work.

We need to observe that $\partial \Omega$, equipped with the metric $d$, is a directionally limited metric space in the sense of Federer [8]. This means that there is an a priori constant $K$ so that, if $S(P, r)$ is a sphere in $\partial \Omega$ of radius $r$, then there can be at most $K$ points in $S(P, r)$ that are spaced at least $r$ apart. This assertion follows because the Cauchy estimates give us an a priori upper bound on the boundary growth of the Szegö kernel, hence a lower bound on the size of the balls $\beta(P, r)$. See [8]for more on the concept of directionally limited metric space.

As a consequence, the Besicovitch covering theorem (and, in particular, the proof of the Besicovitch covering theorem) is valid on $\partial \Omega$ with the metric and balls as indicated, see Section 3 below. ${ }^{1}$ So we know that, if $B(P, r)$ is a fixed ball in $\partial \Omega$, then there are at most $K^{\prime}$ pairwise disjoint balls $B\left(P_{j}, r\right)$ which touch $B(P, r)$. By the triangle inequality, all these balls are contained in $B(P, 3 r)$. Further, if $x$ is a point of $B(P, 3 r)$ that is not contained in $B(P, r)$ nor in any $B\left(P_{j}, r\right)$ then $B(x, r)$ will intersect one of those balls. As a result,

$$
B(P, 3 r) \subseteq \cup_{j=1}^{K^{\prime}} B\left(P_{j}, 2 r\right) .
$$


We see therefore that

$$
\mu\left(B(P, 3 r) \leq\left[\sum_{j=1}^{K^{\prime}} \mu\left(B\left(P_{j}, 2 r\right)\right)\right] .\right.
$$

This is a version of property (b). Thus, we have:

\section{Modified axioms for a space of homogeneous type.}

(a) $0<\mu(B(P, r))<\infty$ for every $P \in X$ and $r>0$;

(b') Given a ball $B(P, r)$, we can find pairwise disjoint balls $B\left(P_{j}, r\right), j=1,2, \ldots, K^{\prime}$, lying in $B(P, 3 r)$, such that

$$
B(P, 3 r) \subseteq \cup_{j=1}^{K^{\prime}} B\left(P_{j}, 2 r\right) \text {; }
$$

(c) there is a $C_{2}>0$ so that, if $B(Q, s) \cap B(P, r) \neq \emptyset$ and $s \geq r$, then $B\left(Q, C_{2} s\right) \supseteq B(P, r)$.

While a bit more technical, these axioms will suffice for the results that we wish to prove below.

So we see that $\partial \Omega$ is a space of homogeneous type with the modified axioms.

3. A covering theorem. Because we have a directionally limited metric space, we can use standard arguments (again see [8]) to prove the following covering lemma of Besicovitch:

Proposition 3.1. There is a number $M>0$ with the following property. Let $B\left(P_{1}, r_{1}\right), B\left(P_{2}, r_{2}\right), \ldots, B\left(P_{k}, r_{k}\right)$ be balls in our metric space $X$ with the property that no ball contains the center of any other (given a covering, it is always possible to extract a refinement with this property). Here $k$ is an arbitrary positive integer. Then our collection of balls can be partitioned into a union of at most $M$ subfamilies so that each subfamily is pairwise disjoint.

Remark 3.2. Our Proposition 3.1 is phrased a bit differently from Federer's Theorem 2.8.14. He formulates the hypothesis in the language of "directionally limited," while we give the condition more explicitly in terms of the centers of the balls. 
Now a standard argument shows that the Hardy-Littlewood maximal function

$$
\mathcal{M} f(x)=\sup _{r>0} \frac{1}{\mu(B(x, r))} \int_{B(x, r)}|f(t)| d t
$$

is weak-type $(1,1)$ (see [6] for this concept). The operator $\mathcal{M}$ is trivially bounded on $L^{\infty}$. By Marcinkiewicz interpolation, we find that $\mathcal{M}$ is bounded on $L^{p}$ for $1<p \leq \infty$.

4. The sub-mean value property. Given a holomorphic function $f$ satisfying a Hardy-type integrability condition on any smoothly bounded domain in $\mathbb{C}^{n}$, it is a standard fact (see [15, page 347]) that the function has a radial boundary limit function $f^{*}$ almost everywhere. So, we can manipulate and compare $f$ with the boundary function $f^{*}$.

The key device for mediating between the holomorphic function $f$ on the interior of $\Omega$ and the boundary function $f^{*}$ is a mean-value or sub-mean value property on discs or polydiscs. This idea is explicated quite clearly in [1] or [15, subsection 8.6]. Here, we develop a slight variant of this idea.

We now note the following. If $z$ is a point of $\Omega$ with Euclidean distance $\delta(z)$ from the boundary, then Cauchy estimates (or the mean value property) show that $|S(z, z)| \leq C \delta(z)^{-n}$. On the other hand, $S$ blows up at an integer rate and that rate must be at least $-n$ because $S$ is a reproducing kernel (a lesser rate would make the Szegö integral a smoothing operator). As a result, it is easy to see that the totally real (or complex normal) extent or size of a ball $B(P, r)$ in the boundary is of size $r$. So, the variation in these balls as we move from boundary point to boundary point is in the complex tangential extent.

Fix $\alpha>1$. On a tubular neighborhood $U$ of $\partial \Omega$, let $\pi: U \rightarrow \partial \Omega$ be the Euclidean normal projection. Given a point $P \in \partial \Omega$ and $\delta>0$ sufficiently small, we consider the set of points

$$
G_{\delta}=G_{\delta}(P) \equiv\{z \in \Omega: \operatorname{dist}(z, \partial \Omega)=\delta, \pi(z) \in B(P, \alpha \delta)\}
$$

(see Section 5 below for further discussion). For each $z \in G_{\delta}$, there is either a complex normal curve $\gamma_{z}$ passing through $z$ and lying in $G_{\delta}$, or perhaps a union of complex normal curves which contains $z$. Now, it is a simple matter to fatten up each such curve into two asymptotically complex one-complex-dimensional half discs $d_{z}^{1}$ and $d_{z}^{2}$. 
This idea is explained cogently in [18]; the proof just depends on the power series. So we have a topological disc $d_{z}$ which is the union of two asymptotically complex half discs. We may take the normal extent of this disclike object to be $\delta / 3$.

A direct calculation shows that, for each such disc $d_{z}$, we may write

$$
|f(z)| \leq \frac{C}{\left(\text { area of } d_{z}\right)} \int_{d_{z}}|f(\zeta)| d A(\zeta) .
$$

Let $\mathcal{D}_{z}$ be the union of all the $d_{z}$ for $z \in G_{\delta}$. The curves $\gamma_{z}$ foliate $G_{\delta}$, and we may use the coarea formula (see [8]) to integrate out over the curves and find that

$$
|f(z)| \leq C \cdot \frac{1}{\left(\text { volume of } \mathcal{D}_{z}\right)} \int_{\mathcal{D}_{z}}|f(\zeta)| d V(\zeta) .
$$

This will be our submean value property which plays the same role as the penultimate line [15, page 359].

5. Boundary behavior. Given a point $P \in \partial \Omega$ and $\alpha>1$, we define an approach region (for calculating the boundary limits of holomorphic functions) as follows. Let $U$ be a tubular neighborhood of $\partial \Omega$, and let $\pi$ be the usual Euclidean normal projection from $U$ to $\partial \Omega$. For each $P \in \partial \Omega$, let $\nu_{P}$ be the Euclidean outward unit normal. Let $\delta_{0}$ be such that the segment from $P-\delta_{0} \nu_{P}$ to $P+\delta_{0} \nu_{P}$ lies in $U$ for each $P \in \partial \Omega$.

Fix $\alpha>1$. Let $G_{\delta}$ be as before. Now set

$$
\mathcal{A}_{\alpha}(P)=\cup_{0<\delta<\delta_{0}} G_{\delta} .
$$

Then $\mathcal{A}_{\alpha}$ will be an approach region at the point $P$.

Example 5.1. Let $\Omega$ be the unit disc in the complex plane. In that context, the Szegö kernel is well known and the balls $\beta$ and $B$ are easily calculated. As a result, we can determine the approach regions $\mathcal{A}_{\alpha}$ to be the traditional nonisotropic approach regions.

Example 5.2. In the case where $\Omega$ is the unit ball in $\mathbb{C}^{n}$, the Szegö kernel is well known to be

$$
S(z, \zeta)=c_{n} \cdot \frac{1}{(1-z \cdot \bar{\zeta})^{n}} .
$$


Thus, the balls $\beta(P, r)$ and $B(P, r)$ are easily calculated to be the usual nonisotropic balls that go back to Koranyi $[\mathbf{1 3}, \mathbf{1 4}]$ and Stein [21]. See also [15]. And the approach region $\mathcal{A}_{\alpha}$ is the nonisotropic approach region originally defined by Koranyi in [13].

Example 5.3. In the case where $\Omega$ is a finite type domain in $\mathbb{C}^{2}$, the balls and approach regions constructed here are just the same as those defined by Nagel/Stein/Wainger in [19].

Now we have the usual isotropic Hardy-Littlewood maximal function $M$ from classical analysis (constructed from nonisotropic boundary balls) and we have the new, nonisotropic maximal function $\mathcal{M}$ that we have constructed here. The two can be used together, as in [1] (see [15] for the details) to obtain the following theorem of Fatou type:

Theorem 5.4. Let $\Omega \subseteq \mathbb{C}^{n}$ be a smoothly bounded, pseudoconvex domain in $\mathbb{C}^{n}$ with the property that $N_{b}$ is pseudolocal. Let $f$ be an $H^{p}$ (i.e., a Hardy space) function on $\Omega, 1 \leq p \leq \infty$. Then, for almost every point $P$ in $\partial \Omega$, the limit

$$
\lim _{\mathcal{A}_{\alpha} \ni z \rightarrow P} f(z)
$$

exists.

This is the main result of the present paper. While the proof follows standard lines, as may be found in [15, Chapter 8], the construction of the ingredients of the proof is new here.

6. Some examples. There is a natural ontology of examples to examine.

First, there is the case of $\Omega$ the unit ball $B$ in $\mathbb{C}^{n}$. Of course, the ball is strongly pseudoconvex, and it is well known that the operator $N_{b}$ is pseudolocal on $B$. So, Theorem 5.4 applies in this case.

The case of smoothly bounded, strongly pseudoconvex domains is similar. The reference [9] is a good source for information about the pseudolocality of $N_{b}$. 
The next level of complexity is finite type domains in $C^{2}$. The study of those domains goes back to the foundational paper [11] of Kohn. And that paper establishes the pseudolocality of $N_{b}$ for such domains.

Finally, we may consider finite type domains in $\mathbb{C}^{n}$. It is known (see [12]) that $N_{b}$ is pseudolocal on domains of finite Kohn ideal type (there are several notions of finite type in higher dimensions, and we cannot explain all the details here). It is also the case that Boas [3] has proved that, on domains of finite D'Angelo type, the Szegö kernel extends smoothly to the boundary off the diagonal.

Thus, we have a wide variety of domains to which our main theorem applies. In practice, as in the case of the finite type domains in $\mathbb{C}^{n}$, it may be rather difficult to calculate the actual geometric shape of the approach regions. The case of finite type domains in $\mathbb{C}^{2}$ can be handled using the rather difficult and delicate estimates of Catlin [5]. So, our approach is broad and abstract, but may be tricky to use in practice.

7. Concluding remarks. The point of view in this paper is to set up the machinery for the study of the boundary behavior of holomorphic functions in an axiomatic fashion. We endeavor to avoid the specific calculations needed to study the disc, the ball, strongly pseudoconvex domains, finite type domains, and so forth. Instead, these particular situations arise as special cases. And other cases, heretofore unexplored, also fit into our paradigm.

We hope in future papers to expand this program to other aspects of complex harmonic analysis.

Acknowledgments. It is a pleasure to thank the referee for many helpful remarks and suggestions. I also thank Harold Boas for several useful references.

\section{ENDNOTES}

1. One of the charming features of the Besicovitch covering theorem is that there is no measure involved in either its statement or its proof.

\section{REFERENCES}

1. S.R. Barker, Two theorems on boundary values of analytic functions, Proc. Amer. Math. Soc. 68 (1978), 48-54. 
2. S.R. Bell, Differentiability of the Bergman kernel and pseudo-local estimates, Math. Z. 192 (1986), 467-472.

3. H. Boas, Sobolev estimates in regular domains, Trans. Amer. Math. Soc. 300 (1987), 109-132.

4. A. Boggess, CR functions and the tangential Cauchy-Riemann complex, CRC Press, Boca Raton, FL, 1991.

5. D. Catlin, Estimates of invariant metrics on pseudoconvex domains of dimension two, Math. Z. 200 (1989), 429-466.

6. R. Coifman and G. Weiss, Analyse harmonique non-commutative sur certains espaces homogenes, Springer Lect. Notes 242, Springer Verlag, Berlin, 1971.

7. F. Di Biase and S.G. Krantz, The boundary behavior of holomorphic functions, Birkhäuser, Boston, 2013.

8. H. Federer, Geometric measure theory, Springer-Verlag, New York, 1969.

9. G.B. Folland and J.J. Kohn, The Neumann problem for the Cauchy-Riemann complex, Princeton University Press, Princeton, NJ, 1972.

10. N. Kerzman, The Bergman kernel function. Differentiability at the boundary, Math. Ann. 195 (1972), 149-158.

11. J.J. Kohn, Boundary behavior of $\bar{\partial}$ on weakly pseudoconvex manifolds of dimension two, J. Diff. Geom. 6 (1972), 523-542.

12. Estimates for $\bar{\partial}_{b}$ on pseudoconvex CR manifolds, Proc. Symp. Pure Math. 43 (1985), 207-217.

13. A. Koranyi, Harmonic functions on Hermitian hyperbolic space, Trans. Amer. Math. Soc. 135 (1969), 507-516.

14. , Boundary behavior of Poisson integrals on symmetric spaces, Trans. Amer. Math. Soc. 140 (1969), 393-409.

15. S.G. Krantz, Function theory of several complex variables, 2nd edition, American Mathematical Society, Providence, RI, 2001.

16. Invariant metrics and the boundary behavior of holomorphic functions on domains in $\mathbb{C}^{n}$, J. Geom. Anal. 1 (1991), 71-98.

17. S.G. Krantz and T. Parsons, Antisocial subcoverings of self-centered covers, Amer. Math. Month. 93 (1986), 45-48.

18. A. Nagel and W. Rudin, Local boundary behavior of bounded holomorphic functions, Canad. J. Math. 30 (1978), 583-592.

19. A. Nagel, E.M. Stein, and S. Wainger, Boundary behavior of functions holomorphic in domains of finite type, Proc. Nat. Acad. Sci. 78 (1981), 6596-6599.

20. , Balls and metrics defined by vector fields, I. Basic properties, Acta Math. 155 (1985), 103-147.

21. E.M. Stein, Boundary behavior of holomorphic functions of several complex variables, Princeton University Press, Princeton, 1972.

Department of Mathematics, Washington University in St. Louis, St. Louis, Missouri 63130

Email address: sk@math.wustl.edu 